\title{
A nonlinear conversion model form ITRFyy to CGCS2000
}

\author{
Fan Wang ${ }^{1 *}$, Peng Zhang ${ }^{1}$, Zhanyi Sun ${ }^{1}$ and Qinglan Zhang ${ }^{1}$ \\ ${ }^{1}$ National Geomatics Center of China, Beijing, 100830
}

KEY WORDS: CGCS2000, ITRF, conversion, nonlinear, S-transformation

\begin{abstract}
:
At present, ITRS series reference frameworks are widely used in the world. The results of GNSS are mostly based on the ITRF framework. Transform from ITRF to CGCS2000 is not easy, which restricts the promotion and use of CGCS2000. The conversion relationship between CGCS2000 and ITRF framework has imminent practical significance. This paper constructs the epoch reduction and frame conversion two-steps model which estimated the nonlinear model to solve the appeal problem. Effective test show that the nonlinear model accesses an improvement in not only precession but also accuracy relative to the tradition model.
\end{abstract}

\section{INTRODUCTION}

The CGCS2000 which was released in 2008 means our national geodetic coordinate system has transformed from reference ellipsoid centric coordinate to geocentric coordinate system, is a landmark in the research and application of geodetic survey in China (Chen, 2008; Yang, 2009; Wei ,2008). It also indicates that our geodetic reference system study has been in line with international practice. CGCS2000 have already made major contributions to our national defence construction. However, how to accurately calculate the results under the instantaneous epoch to the CGCS2000 framework has always been a bottleneck restricting the promotion and application of CGCS2000. This paper uses the epoch reduction and frame conversion two-steps method to solve the appeal problem.

\section{THE NONLINEAR EPOCH CALCULATED MODEL}

The coordinates of the transient epoch under the ITRFyy framework first should been calculated to 2000.0 epoch. Conversion parameters based on a specific epoch are usually calculated using the model which only considers the linear motion of the site.

(1)

$$
\begin{aligned}
& \text { Where } \mathbf{t}_{\mathbf{i}} \text { is transient time } \\
& \mathbf{t}_{\mathbf{0}} \text { is origin time } \\
& \text { is the velocity of the station }
\end{aligned}
$$

However a large number of international studies have shown that the motion of the site is nonlinear, including not only the periodic motion of the annual and semi-annual periodicity, but also the jumps caused by tectonic motion such as large earthquakes, and the post-seismic deformation. Therefore, this paper adopts a model that takes into account nonlinear motion. The conversion model to the three position components is as follows:

$$
y\left(t_{i}\right)=y\left(t_{0}\right)+v\left(t_{i}-t_{0}\right)+c+p \log \left[1+\frac{t_{i}-t_{e q}}{\tau}\right]+\sum_{i}\left[S_{i} \sin \left(\varpi_{i} t\right)+C_{i} \cos \left(\varpi_{i} t\right)\right]
$$

It can be seen that the above model is mainly composed of three parts: the influence of tectonic movement such as earthquake, the long-term tectonic velocity term and the anniversary term. In order to accurately determinate this three models, the observation data from 2011-2017 of 410 national GNSS stations which located in our mainland were used. We considering the nonlinear and liner characteristics simultaneously, which means establish the above function model based on each GNSS station's time series, using parameter estimation method quantitatively estimated co-seismic, post-seismic, velocity and annual and semi-annual periodicity.

\section{GNSS data process method}

1) Daily process

In order to eliminate the influence of the inconsistency of the model and processing strategy in data processing, the same model and method are used to uniformly process the above data using GAMIT/GLOBK (Herring, 2002) software. The processing of the GPS carrier data is performed in a 24-hour period, using a double-difference mode and a satellite orbit relaxation solution. To reduce calculation time, we divides the observatory into five sub-areas according to its geographical location, every partitions are processed separately. Each partition is bound together in the next step by public parameters. The obtained single-day relaxation solutions include the station position, the estimate of the satellite orbital lamp parameters, and the variance-covariance matrix.

2) Multi session adjustment

The above daily relaxation solutions are combined through public estimates to obtained a total solution. Further, the transform parameters relative to the ITRF2014 are estimated by the globally distributed base stations which are included in the solution, and finally the single day non-reference solutions are converted to the ITRF2014 through the obtained 7 parameters. So we have got the continuous station position time series under the ITRF2014 framework.

Where is the co-seismic deformation is the post-seismic deformation

\footnotetext{
* Corresponding author: Eamil: wangfan@ngcc.cn
} 

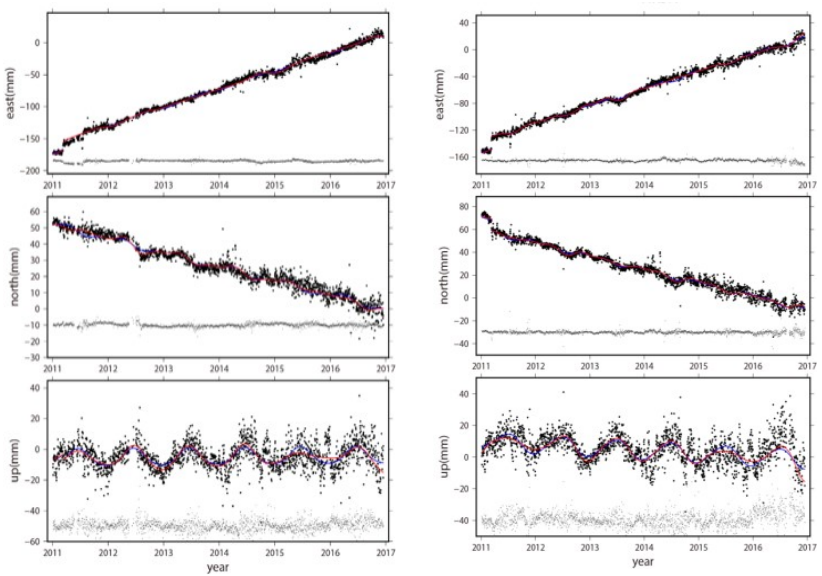

Figure 1. time series of JIXN(left) and HRBN(right) station.

\subsection{Periodic model of GNSS reference station}

We use the spectral analysis method to study the time series, aimed to detected whether the time series contain some other periodic signals, it can also verify the geophysical models have already been deducted in data process or not. The results of spectral analysis show that the most significant signal in the time series of horizontal direction is the annual period (see Figure 2, Figure 3), the semi-annual period followed.

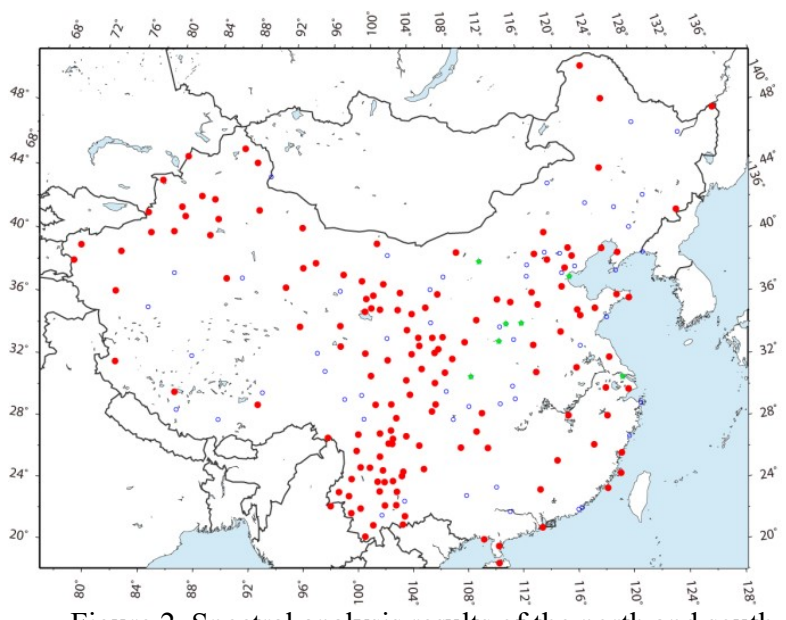

Figure 2. Spectral analysis results of the north and south direction. (Red circle means the main period is annual, blue circle shows the main period is semi-annual, and green circle represents the main period is other term)

\subsection{Velocity model of GNSS reference station}

Based on the above model, we get the velocity field of Chinese mainland (see Fig 4), the average possible error of horizontal velocity is $\pm 0.3 \mathrm{~mm}$, vertical direction is about $\pm 0.5 \mathrm{~mm}$.

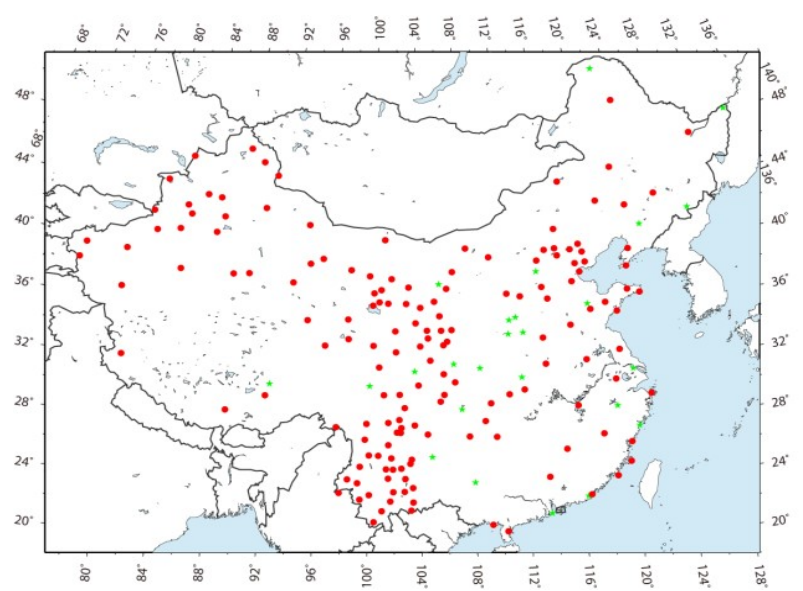

Figure 3. Spectral analysis results of the east and west direction. (the same as Fig 2)

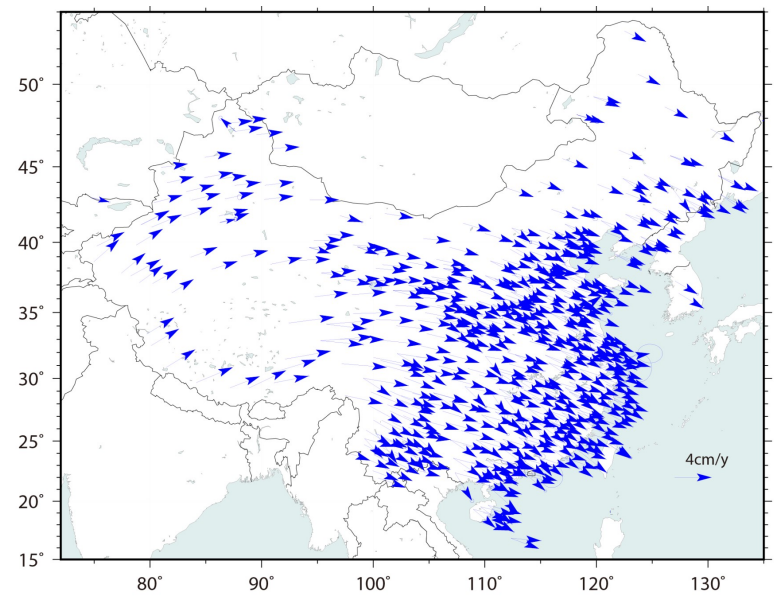

Figure 4. Velocity field of Chinese mainland (relative to ITRF2014)

\section{METHOD FOR REFERENCE TRANSFORMATION}

Tight constraint method and S-transformation method are the two widely used methods for frame transformation.

\subsection{Tight constraint method:}

A wide range of papers has been published on the concept of the free network and optimal methods of computing a set of coordinates from a singular normal equation. The set of constraints usually added to a free-network normal equation by tight constrained method (Mittermyer 1972; Perelmuter 1979; Blaha 1982;Dermanis 1994a;Xu 1997). The tight constraint is used in the situation that absence of datum, some stable stations are generally selected as the core station, and the value of the coordinate and velocity under a certain reference frame is taken as true value. It need high accuracy of the core stations' coordinates. Constrained least squares estimates can be implemented with Lagrangian multipliers. Although it is very effective to solve the rank-deficient problem of the normal equation, however it will cause the geodetic control network be deformed, means will generate bias for the coordinate of the stations that located far away from the core station. 


\subsection{S-transformation method:}

S-transformation (also be called Helmert transformation) method is introduced by Baarda in the early 1950s. A lot of authors have contributed to this subject since then, such as Mierlo (Mierlo ,1980), who discussed free-networks analysis and the answer that can be given to the rank deficiency of the normal equations by S-transformations. Several researchers Teunissen, 1985; Koch, 1987; Crosilla et al. 1989; Xu, 1997) have delved more deeply into the problem, each adding their own contribution. The standard relation of transformation between two reference systems is an Euclidian similarity of seven (or fourteen) parameters: three translations, one scale factor, and three rotations designated respectively.

\subsection{Bias caused by the two methods}

In order to analyze the difference between the tight constraint method and the S-transformation method, we used the data of 1900 GNSS reference stations that located in China during August 1st to 31st, 2014 aimed to verify the different methods. After obtained the daily loosely solution per day (e.g. in which stations' positions and velocities are constrained to a priori values with for positions and $\delta \mathbf{I} 10 \mathrm{~cm} / \mathrm{yr}$ for velocities), the relax network were aligned to ITRF2008 by using the two methods which are described above. Then we compared the coordinate results. The difference in coordinates are shown in Figure 2-10.

It can be seen that the tight constraint method and the Stransformation method are quite different, for $\mathrm{X}$ direction, $50 \%$ stations' bias are in $1 \mathrm{~cm}, 90 \%$ stations's bias are in $5 \mathrm{~cm}$, similar for the $\mathrm{Y}$ direction. The bias of the $\mathrm{Z}$ direction are much bigger, especially some stations have already up to $10 \mathrm{~cm}$. Therefore, we use the S-transformation method as the framework transformation.
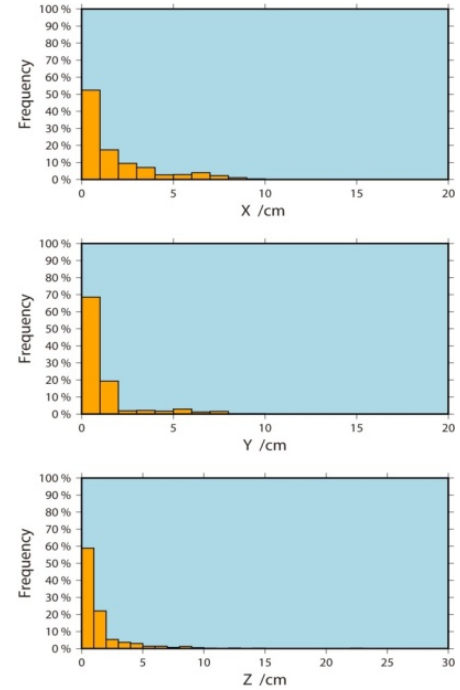

Figure 5. bias of the XYZ directions

Typically, the ITRF conversion uses 14 conversion parameters (ie, the rate of seven conversion parameters plus seven conversion parameters). The conversion between different ITRF frameworks is established by 14 parameters. The conversion parameters are given by IERS (https://www.iers.org/IERS/EN/ DataProducts/ITRF/itrf.html) , as shown in the following table.
Table 1、 transform parameter

\begin{tabular}{|c|c|c|c|c|c|c|c|c|}
\hline & $\begin{array}{l}\mathrm{Tx} \\
/ \mathrm{cm}\end{array}$ & $\begin{array}{l}\text { Ty } \\
/ \mathrm{cm}\end{array}$ & $\begin{array}{l}\mathrm{Tz} \\
/ \mathrm{cm}\end{array}$ & $\begin{array}{l}\mathrm{D} \\
/ \mathrm{ppb}\end{array}$ & $\begin{array}{l}\mathrm{Rx} \\
0.001\end{array}$ & $\begin{array}{l}\text { Ry } \\
0.001\end{array}$ & $\begin{array}{l}\mathrm{Rz} \\
0.001\end{array}$ & epoch \\
\hline & $\mathrm{cm} / \mathrm{a}$ & $\mathrm{cm} / \mathrm{a}$ & $\mathrm{cm} / \mathrm{a}$ & $\mathrm{ppb} / \mathrm{a}$ & $\begin{array}{l}0.001 \\
\text { "/a }\end{array}$ & $\begin{array}{l}0.001 \\
1 / \mathrm{a}\end{array}$ & $\begin{array}{l}0.001 \\
1 / \mathrm{a}\end{array}$ & \\
\hline \multirow{2}{*}{$\begin{array}{l}\text { ITRF } \\
2014\end{array}$} & 7.4 & -0.5 & -62.8 & 3.80 & 0.00 & 0.00 & 0.26 & \multirow[b]{2}{*}{2010.0} \\
\hline & 0.1 & -0.5 & -3.3 & 0.12 & 0.00 & 0.00 & 0.02 & \\
\hline \multirow{2}{*}{$\begin{array}{l}\text { ITRF } \\
2008\end{array}$} & 4.8 & 2.6 & -33.2 & 2.92 & 0.00 & 0.00 & 0.2 & \multirow[b]{2}{*}{2000.0} \\
\hline & 0.1 & -0.5 & -3.2 & 0.09 & 0.00 & 0.00 & 0.02 & \\
\hline \multirow{2}{*}{$\begin{array}{l}\text { ITRF } \\
2000\end{array}$} & 0.67 & 0.61 & -1.85 & 1.55 & 0.00 & 0.00 & 0.00 & \multirow{2}{*}{1997.0} \\
\hline & 0.0 & -0.06 & -0.14 & 0.01 & 0.00 & 0.00 & 0.02 & \\
\hline
\end{tabular}

The transformation model is

$\left[\begin{array}{c}\mathrm{X}_{s} \\ \mathrm{Y}_{s} \\ \mathrm{Z}_{s}\end{array}\right]_{\text {CGCS } 2000}=\left[\begin{array}{c}\mathrm{X} \\ \mathrm{Y} \\ \mathrm{Z}\end{array}\right]_{\text {CGCS } 2000}+\left[\begin{array}{c}\mathrm{T}_{x} \\ \mathrm{~T}_{y} \\ \mathrm{~T}_{\mathrm{z}}\end{array}\right]+\left[\begin{array}{ccc}D & -e_{z} & e_{y} \\ e_{z} & D & -e_{x} \\ -e_{y} & e_{x} & D\end{array}\right]\left[\begin{array}{l}\mathrm{X} \\ \mathrm{Y} \\ \mathrm{Z}\end{array}\right]_{\text {ITRFx }}$

\section{PRECESSION ANALYSIS OF THE CONVERSION MODEL}

In order to verify the validity of the transformation model established in this paper, 21 benchmarks with CGCS2000 true coordinate were selected for comparison. Test 1: When epochs are reduced, regardless of the impact of major earthquakes, annual events, etc., linear models are used to describe site position changes. Test 2: Estimate the impact of major earthquakes and annual events, using a nonlinear model to describe site position changes. The difference between the CGCS2000 coordinate results and the true values obtained by the two tests are shown in the Fig 6 and Fig7.

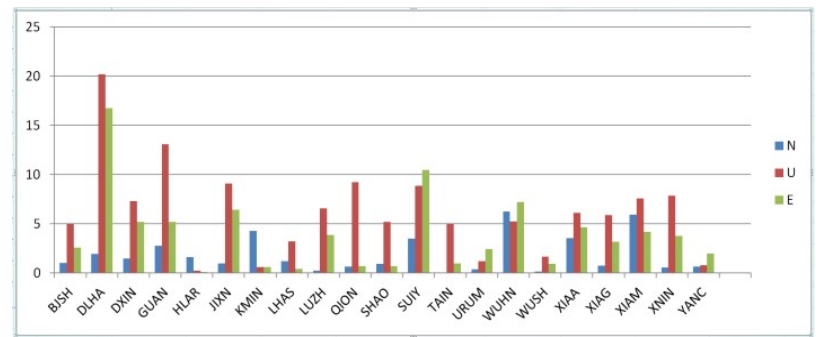

Figure 6. Coordinate differences of Test 1.(unit is $\mathrm{cm}$ )

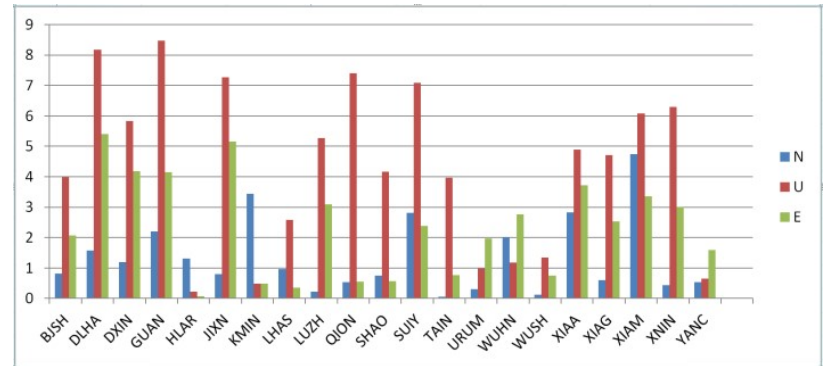

Figure 7. Coordinate differences of Test 2.(units is $\mathrm{cm}$ )

It reveals that Test 1: horizontal differences are mostly within 2-3 cm, but elevation differences are large. The largest GUAN, WUHN had up to DM magnitude, HAIK and XIAA are close to $\mathrm{dm}$. Test 2: The horizontal differences are mostly within $1 \mathrm{~cm}$, 
the elevation differences are about $50 \%$ within $5 \mathrm{~cm}$, and the largest GUAN and DLHA are about $8 \mathrm{~cm}$. Test 2 had obviously improved the accuracy compared with test 1 . At the same time, it proves that the transformation model constructed in this paper has very high accuracy.

\section{CONCLUSIONS}

We create a model which consider the nonlinear term of stations' time series to convert the transient epoch results under ITRFyy to CGCS2000. In the first step, coordinates of transient epoch are converted to the 2000.0 epoch, during this period we not only estimate the velocity term but also the nonlinear terms such as co-seismic, post-seismic displacement, load effect and son on. In the second step, we transform the coordinates of ITRFyy to CGCS2000 by using a S-transformation method. Then, we test the precession of the new model by comparing the coordinates generated from this model and the true values of 21 stations. The horizontal differences are mostly within $1 \mathrm{~cm}$, the elevation differences are about $50 \%$ within $5 \mathrm{~cm}$, and the largest GUAN and DLHA are about $8 \mathrm{~cm}$. It is obvious that the new model is more accurate compare with the tradition model, meaning that the model constructed in this paper has very high accuracy and precession.

\section{ACKNOWLEDGEMENTS}

This work was supported by National Natural Science Foundation of China (41304008).

\section{REFERENCES}

Chen Junyong., 2008: Chinese Modern Geodetic DatumChinese Geodetic Coordinate System 2000(CGCS2000) and Its Frame. ACTA GEODAETICA et CARTOGRAPHICA AINICA., 37(3):269-271

Yang Yuanxi, 2009: China Geodetic Coordinate System 2000, Bulletion of Science, 54(16):2271-2276

Wei Ziqin, 2008: China Geodetic Coordinate System 2000, Journal of Geodesy and Geodynamics, 06:1-5

TA Herring, R W King, S C McClusky, 2009, GAMIT Reference Manual: GPS Analysis at MIT Release 102, http://www.gps.mit.edu.

TA Herring, R W King, S C McClusky, 2009, GLOBK Reference Manual: GPS Analysis at MIT Release 102, http://www.gps.mit.edu.

Mittermayer E, 1972: Equvalence of estimable quantities and invariants in geodetic networks, Bull Geod ,46:281-290

Perelmuter A,1979: Adjustment of free networks, Bull Geod ,53:281-290

Blaha G, 1982: Free networks: minimum norm solution as obtained by the inner adjustment constraint method. Bull Geod ,56:209-219

Dermanis A,1994: Free networks solutions with the Direct Linear Transformation (DLT) method. ISPRS J Photogram Remsens 49:2-12
Xu P, 1997: A general solution in geodetic non-linear rank defetct models. Boll Geod Sci Aff, 1:1-25

Revised May 2019 